\title{
Attitude Control Method of Spin-Stabilized Satellite Base on Equiangular Precession
}

\author{
Yonggang Li ${ }^{a}$, Jiping Ren ${ }^{b}$, Libing Guo ${ }^{c}$, Wen Mao ${ }^{d}$, Yi Wang ${ }^{e}$ \\ China Satellite Maritime Tracking and Controlling Department \\ alee@163.com, bjiping_ren@163.com, cguoguo_leo@163.com, dwen_mao@163.com, \\ ewang_yi@163.com
}

\begin{abstract}
Attitude control is a basic task of a spin-stabilized satellite control. There are many kinds of attitude control methods, this paper does research for equiangular precession method. The paper first introduced the basic principle of equiangular precession method, and then gave out a detailed calculation method of attitude control quantity, which included theory executing angular, executing times and time delay, finally attitude maneuver process were simulated. The simulation result shows that the computed result of attitude control quantity is correct, the forecasts for control process are essentially the same as practice. The method has been applied to satellite launch mission.
\end{abstract}

Keywords: Spin-stabilized Satellite; Attitude Control; Equiangular Precession; Theory Executing Angular; Executing Times.

\section{Introduction}

As the needs of the project, spin-stabilized satellites need to establish a certain spin axis orientation, which requires the spin axis orientation to be controlled in space. According to the projected trajectory on the celestial sphere of the spin axis direction in the process of attitude maneuver, the control method can be divided into equiangular precession, large circular arc method, the latitude and longitude method [1], the control modes are all spin synchronized. As the impulse of a pulse is small, the spin axis direction is consistent with the angular momentum during the attitude maneuver. The control path of the large circular arc method is the shortest and most fuel-optimal control, but the jet phase value is correlative with the real attitude, and need to recalculate the phase angle before each jet, so the implementation is more complex. The equiangular precession method is the most convenient for engineering implementation [2].

\section{The basic principles and methods of attitude control}

\subsection{Basic concepts and principles.}

The attitude control of the spin satellite refers to use the suitable control law to precess the initial attitude to the end attitude in the control of satellite coordinates. Actually, attitude control method is jet phase and arc calculation. Precession angle is that each engine jet phase is constant, that is to say, the angle $\beta$ of the celestial sphere (Figure 1) between the track direction and the longitude is a constant value. Therefore, before the spin angle attitude controlling of satellites, needs to ascertain the executive pulse width [3], and obtain the angular surface, the execution frequency of the pulse, and the total delay through computation ( called the attitude control volume ).

The control coordinate includes the sun reference system and the earth reference system. $A_{0}$ and $A_{f}$ are the initial attitude and the end attitude respectively of the control coordinate system as shown in Figure 1, A is any one time attitude. Attitude vector denotes the longitude angle $\Psi(-\pi<\Psi \leq \pi)$ and the latitude angle $\theta(0 \leq \theta \leq \pi)$ of the control coordinate system.

The benchmark of the sun reference control system $\left(\mathrm{OXX}_{S} \mathrm{Y}_{\mathrm{S}} \mathrm{Z}_{\mathrm{S}}\right)$ is the sun with the origin at the center of mass of the satellite, $\mathrm{Z}_{\mathrm{S}}$ axis points to the sun, $\mathrm{X}_{\mathrm{S}}$ axis is perpendicular to the $\mathrm{Z}_{\mathrm{S}}$ axis in a longitude plane, shows as in Figure 2. $\mathrm{OX}_{\mathrm{I}} \mathrm{Y}_{\mathrm{I}} \mathrm{Z}_{\mathrm{I}}$ is the geocentric inertial, $\boldsymbol{\alpha}_{s}$ and $\boldsymbol{\delta}_{s}$ are the sun right ascension and declination. 
Satellite initial attitude and four engine ignition attitude are the geocentric inertial attitude measurement obtained, and it is necessary to convert the geocentric inertial attitude to the control frame in the calculation of the satellite precession angle.

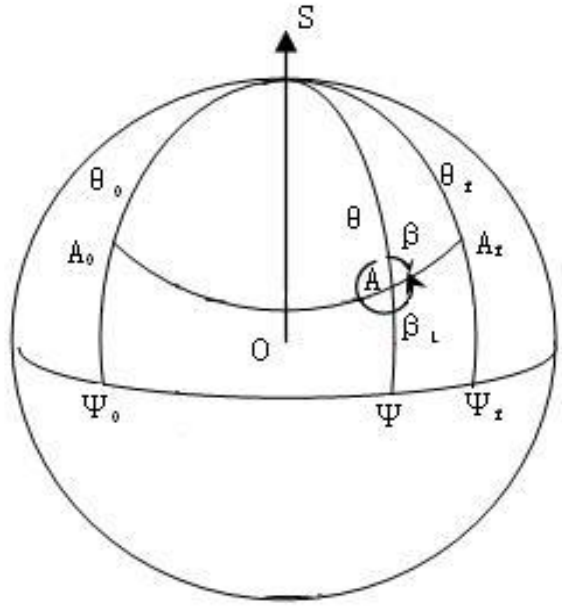

Figure 1 Attitude celestial sphere

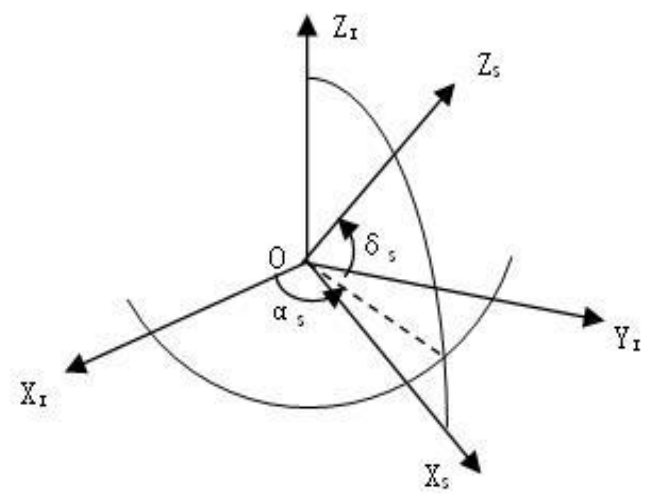

Figure 2 sun reference system

\subsection{Attitude conversion.}

The attitude transformation matrix of the inertial to the sun reference system for the ASI:

$$
\mathbf{A}_{\mathbf{S I}}=\mathbf{R}_{y}\left(\pi / 2-\delta_{S}\right) \mathbf{R}_{Z}\left(\alpha_{S}\right)=\left[\begin{array}{ccc}
\sin \delta_{S} \cos \alpha_{S} & \sin \delta_{S} \sin \alpha_{S} & -\cos \delta_{S} \\
-\sin \alpha_{S} & \cos \alpha_{S} & 0 \\
\cos \delta_{S} \cos \alpha_{S} & \cos \delta_{S} \sin \alpha_{S} & \sin \delta_{S}
\end{array}\right]
$$

$\alpha_{s}$ and $\delta_{s}$ are the sun right ascension and declination.

The attitude transformation matrix of the inertial to the earth reference system for the $\mathrm{A}_{\mathrm{EI}}$ :

$\mathbf{A}_{\mathbf{E I}}=\mathbf{R}_{x}(-\pi / 2) \mathbf{R}_{z}(\pi / 2) \mathbf{R}_{z}(u) \mathbf{R}_{x}(i) \mathbf{R}_{z}(\Omega)$

$$
=\left[\begin{array}{ccc}
-\sin u \cos \Omega-\cos u \cos i \sin \Omega & -\sin u \sin \Omega+\cos u \cos i \cos \Omega & \cos u \sin i \\
-\sin i \sin \Omega & \sin i \cos \Omega & -\cos i \\
-\cos u \cos \Omega+\sin u \cos i \sin \Omega & -\cos u \sin \Omega-\sin u \cos i \cos \Omega & -\sin u \sin i
\end{array}\right]
$$

$i$ is orbital inclination, and $\Omega$ is ascending node, $u$ is the satellite position starting from the ascending node; $\mathrm{R}_{x}, \mathrm{R}_{y}$ and $\mathrm{R}_{z}$ is the rotation matrix [4]. Calculates the angle $\Psi$ and the colatitude $\theta$ given that right ascension and declination of the attitude according to the equation (3).

$$
\psi= \begin{cases}\operatorname{tg}^{-1}\left(p_{y} / p_{x}\right) & p_{x}>0 \\ \pi / 2 & p_{x}=0 \\ \operatorname{tg}^{-1}\left(p_{y} / p_{x}\right)+\pi & p_{x}<0, p_{y} \geq 0 \\ \operatorname{tg}^{-1}\left(p_{y} / p_{x}\right)-\pi & p_{x}<0, p_{y}<0\end{cases}
$$

$\theta=\cos ^{-1}\left(p_{z}\right)$ 
$\left[\begin{array}{l}p_{x} \\ p_{y} \\ p_{z}\end{array}\right]=\mathbf{A}_{\mathbf{I}} \vec{P}_{I}=\mathbf{A}_{\mathbf{I}}\left[\begin{array}{l}\cos \alpha \cos \delta \\ \sin \alpha \cos \delta \\ \sin \delta\end{array}\right]$

$\alpha, \delta$ are the satellite attitude of ascension and declination; $\vec{P}_{I}$ is satellite attitude vector; $\mathbf{A}_{\mathbf{I}}$ is attitude transformation matrix in the sun reference for $A_{S I}$, and in the Earth reference system for $A_{E I}$.

\subsection{Precession angle and satellite phase control angle.}

Figure 3 shows the inclination of the satellite attitude on the right precession, which is a part of Figure 1 attitude on the celestial sphere [5].

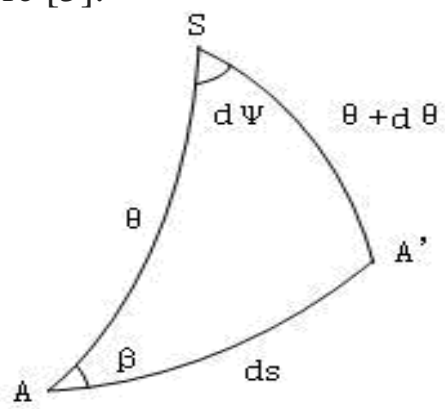

Figure 3 Satellite attitude precession to right

In the spherical triangle SAA', because ds is very small, it can be seen as an arc. It can be available by the sine and cosine formula [6].

$$
\begin{aligned}
& \sin d s / \sin d \psi=\sin (\theta+d \theta) / \sin \beta \\
& \cos (\theta+d \theta)=\cos \theta \cos d s+\sin \theta \sin d s \cos \beta
\end{aligned}
$$

When the ds is small, $d \psi$ and $d \theta$ are small, spreads the equation (4) and (5), linearization derived formula (6) and (7).

$$
\begin{aligned}
& d s / d \psi=\sin \theta / \sin \beta \\
& d s=-d \theta / \cos \beta
\end{aligned}
$$

Brings formula (7) into equation (6):

$$
d \psi=-\operatorname{tg} \beta d \theta / \sin \theta
$$

Integral calculus both sides of the equation:

$$
\Delta \psi=\psi_{f}-\psi_{0}=\operatorname{tg} \beta \ln \left(\frac{\operatorname{tg}\left(\theta_{0} / 2\right)}{\operatorname{tg}\left(\theta_{f} / 2\right)}\right)
$$

Therefore,

$$
\begin{aligned}
& \beta=c t g-1\left[\frac{1}{\Delta \psi} \ln \left(\frac{\operatorname{tg}\left(\theta_{0} / 2\right)}{\operatorname{tg}\left(\theta_{f} / 2\right)}\right]\right. \\
& \Delta \psi= \begin{cases}\psi_{e} & \left|\psi_{e}\right| \leq \pi \\
\psi_{e}-2 \pi & \pi<\psi_{e} \leq 2 \pi \\
\psi_{e}+2 \pi & -2 \pi \leq \psi_{e}<-\pi\end{cases}
\end{aligned}
$$

So the satellite phase control angle $\beta_{L}$ is,

$$
\beta_{L}= \begin{cases}2 \pi-\beta & \Delta \psi \geq 0 \\ \beta & \Delta \psi<0\end{cases}
$$

\subsection{Theory execution angle.}

When using the axial engine controlling, in the direction of the spin axis, the direction of the angular momentum coming from axial thrust lags jet phase at 90 degrees, the jet phase angle $\beta^{*}$ is calculated according to the following conditions. When using the sun as a reference point:

$$
\beta^{*}=\beta_{L}-\pi / 2+\beta_{a}
$$

\subsection{Pulse execution times and delay}

(1) Pulse execution times

When $\theta_{0} \neq \theta_{f}$, calculating the control arc length $S$ in calculus of the formula (7). 


$$
S=\left|\left(\theta_{0}-\theta_{f}\right) / \cos \beta\right|
$$

When $\theta_{0}=\theta_{f}, \theta$ is a constant, and $\beta=\pi / 2$, calculating the control arc length $S$ by the formula (6).

$$
S=\left|\left(\psi_{f}-\psi_{0}\right) \sin \theta_{0}\right|
$$

The momentum precession arc $\Delta S$ of a pulse could be available based on the derivation of theoretical mechanics.

$$
\Delta S=\frac{\Delta I L \cos \xi}{J_{z} \omega_{0}}
$$

$\Delta I$ is rotation impulse; $L$ is arm of force; $\xi$ is axis nozzle angle; $J_{z}$ is inertia around the spin axis; $\omega_{0}$ is satellite speed.

We can get the total number of theoretical control jet from the control pulse arc $S$ and $\operatorname{arc} \Delta S$ of a pulse. Where the symbol [| |] indicates rounding.

$$
N_{d}=[|S / \Delta S|]
$$

The number of actual control theory calculating includes unsteady $\left(N_{d}<100\right)$ and steady state $\left(N_{d} \geq 100\right.$ ). The actual control number of the steady state is calculated as follows. Firstly, calculate the fuel $G$ of the tank.

$$
G=\gamma\left[V-V_{0} T P_{0} /\left(T_{0} P\right)\right]
$$

Where $r$ is the proportion of hydrazine; $V$ is system tank volume; $V_{0}$ is system air volume; $T_{0}$ is the temperature when filling nitrogen; $T$ is the temperature of hydrazine bottle measured; $P_{0}$ is nitrogen pressure when filling; $P$ is the pressure of hydrazine bottle measured.

Then, set the average pressure initial value $\bar{P}=P$, the fuel of attitude maneuver $\Delta G$ can be obtained by the formula (14), $I_{s}$ is specific impulse, $G_{0} 、 G_{1} 、 G_{2} 、 G_{3}$ are calculation coefficient of continuous impulse provided by satellite engineering constants.

$$
\begin{aligned}
& I_{s}=G_{0}+G_{1} \bar{P}+G_{2} \bar{P}^{2}+G_{3} \bar{P}^{3} \\
& \Delta G=\left(\sum \Delta I\right) / I_{s}=J_{z} \omega_{0} S /\left(L \cos \xi I_{s}\right)
\end{aligned}
$$

The remaining fuel and pressure after attitude maneuver:

$$
\begin{gathered}
G_{f}=G-\Delta G \\
P_{f}=\left(T / T_{0}\right) P_{0} V_{0} /\left(V-G_{f} / \gamma\right)
\end{gathered}
$$

Therefore, the average pressure for attitude maneuver:

$$
\bar{P}=\left(P+P_{f}\right) / 2
$$

Return to the formula (18), go to iterative calculate until the difference of $\bar{P}$ twice is less than a smaller value. The average steady rotation impulse $\Delta \bar{I}$ of attitude maneuver can be calculated with $\bar{P}$ the last iteration calculate.

$$
\Delta \bar{I}=\left[C_{01}+C_{11} \bar{P}+C_{21} \bar{P}^{2}+G_{31} \bar{P}^{3}\right]
$$

$C_{01}, C_{11}, C_{21}, C_{31}$ are calculation coefficient of rotation impulse provided by satellite engineering constants. Finally, the actual control number $N$ of pulses:

$$
\begin{aligned}
& N=[|\tilde{N}|] \\
& \tilde{N}=S / \Delta S=S J_{z} \omega_{0} /(\Delta \bar{I} L \cos \xi)
\end{aligned}
$$

(2) Total Delay. The total delay refers to the sum of equipment delay, space delay and thrust center delay. device delay includes the delay of the telecontrol device sending instruction and the satellite remote terminal received instruction. Since the control pulse jet will persist for some time, the time from the leading edge to the equivalent moment is called thrust center delay. Space delay includes satellite recovery and path delay from the station to the satellite space. Therefore, the total delay for the start-up control time:

$$
T_{d}=T_{t}+2 \rho / C+T_{p}
$$


Where $\rho$ the ramp distance from the satellite to stations is, $C$ is speed of light.

\section{Application Analysis}

\subsection{Simulation.}

Using the engineering parameters of a spin-satellite, selects the sun control reference, the control pulse width is 150 , the orbit is shown in Table 1 , the control value is shown in Table 2, the temperature and pressure parameters is shown in Table 3, the start control time was at 14:10:18 on December 23 2008, the calculation results of the attitude control are shown in Table 4.

Table 1 Orbital elements

\begin{tabular}{|c|c|}
\hline Epoch & $13: 20: 25$ on the December 23,2008 \\
\hline Orbital Axis $a$ & $24239.099 \mathrm{~km}$ \\
\hline Eccentricity $e$ & 0.728482 \\
\hline Orbital inclination $i$ & $24.091252^{\circ}$ \\
\hline Ascend Node $\Omega$ & $95.717799^{\circ}$ \\
\hline Perigee $\omega$ & $180.728361^{\circ}$ \\
\hline Mean Anomaly $M$ & $139.849719^{\circ}$ \\
\hline
\end{tabular}

Table 2 Control quantity

\begin{tabular}{|c|c|c|}
\hline & Initial attitude & End attitude \\
\hline Ascension $\alpha$ & $3.606^{\circ}$ & $179.65^{\circ}$ \\
\hline Declination $\delta$ & $-24.08^{\circ}$ & $-40.24^{\circ}$ \\
\hline Speed $\omega$ & $43.0 \mathrm{rpm} / \mathrm{min}$ & $/$ \\
\hline
\end{tabular}

Table 3 Temperature and pressure before control

\begin{tabular}{|c|c|c|c|}
\hline A1 system temperature & $21.2^{\circ}$ & A2 system temperature & $20.9^{\circ}$ \\
\hline B1 system temperature & $21.1^{\circ}$ & B2 system temperature & $21.0^{\circ}$ \\
\hline A system fuel & $29.2 \mathrm{~kg}$ & B system fuel & $29.7 \mathrm{~kg}$ \\
\hline A system pressure & $16.6 \mathrm{~kg} / \mathrm{cm}^{2}$ & B system pressure & $17.6 \mathrm{~kg} / \mathrm{cm}^{2}$ \\
\hline
\end{tabular}

Table 4 Computed result of attitude control quantity

\begin{tabular}{|c|c|}
\hline The value of attitude control & Calculation results \\
\hline Execution angle $\beta^{*}$ & $27.8^{\circ}$ \\
\hline The number of pulses N & 767 times \\
\hline The total delay Td & $639.3 \mathrm{~ms}$ \\
\hline
\end{tabular}

\subsection{Simulation Control.}

Carries on attitude controlling using calculated results in Table 4. After attitude controlling, the ascension is 180.3 degrees, the declination is -40.6 degrees. The control error of ascension is 0.65 degrees, and the control error of declination is 0.36 degrees, meets the control requirements of the transfer orbit attitude maneuver.

\section{Conclusion}

In the processing of actual spin-satellite attitude controlling, because the attitude control parameter calculation process exists various errors, it will affect the actual control. In order to improve the accuracy of the attitude control parameter needs to demarcate the factor of the engine in the actual engineering applications. Execution angle and times correction is an effective way to improve the precision of attitude control. The methods of spin-satellite attitude controlling were applied in some satellites launch in this paper, and have achieved good results.

\section{References}

[1] Huang Fu-ming, Hao He-nian. Spacecraft Flight Control and Simulation [M] Beijing; National Defense Industry Press, 2004:73-80. 
[2] Tu Shan-cheng, Zou Guang-rui. Satellite Attitude Dynamics and Control (2) [M] Beijing; Aerospace Press, 1998:24-25.

[3] Chen Fangyun. Satellite Control Manual [M] Beijing; Science Press, 1993:400-401.

[4] Zhu Min-cai, Tang Geshi. Space Orbit Determination, Orbit control and Mission planning [M] Beijing; National Defense Industry Press, 2007:18-19.

[5] Liu Lin. Artificial Earth Satellite Orbital Mechanics [M] Beijing; Higher Education Press, 1992

[6] [6]Bai Wen-liang, Gu Bing-jun. Spin-Satellite Maritime Tracking and Control Attitude Control and Implementation [J]. Shanghai Aerospace, 2007, 24 (05): 58-61. 